\title{
Liver abscess caused by Actinomyces odontolyticus, Abiotrophia species, Haemophilus parainfluenzae, Streptococcus anginosus i Streptococcus intermedius - case report
}

\author{
GORDANA CAVRIĆ $C^{1}$ KHALED NASABAIN ${ }^{2}$, KLARA JURIĆ ${ }^{3}$ INGRID PRKAČIN ${ }^{1}$, \\ DUBRAVKA BARTOLEK HAMP ${ }^{4}$, MATEA BOGDANOVIĆ DVORŠČAK \\ ${ }^{1}$ Department of Internal Medicine, University Hospital Merkur, Zagreb, Croatia \\ ${ }^{2}$ Centre Hospitalier de l'Ouest Vosgien, Pole mére-enfant, Neufchâteau, France \\ ${ }^{3}$ Department of Internal Medicine, University Hospital Dubrava, Zagreb, Croatia \\ ${ }^{4}$ Department of Anaesthesiology, Reanimation and Intensive Care, General Hospital Dubrovnik, Croatia \\ ${ }^{5}$ Department of Anaesthesiology, Reanimation and Intensive Care, University Hospital Merkur, Zagreb, Croatia
}

Corresponding author: Gordana Cavrić, Zajčeva 19, University Hospital Merkur, Zagreb, Croatia,

Phone: +38512253207, Fax: +38512431393,E-mail: gordana.cavric1@gmail.com

\section{ABSTRACT}

We present a case of a 78-year-old female patient who was admitted to the medical intensive care unit because of high fever, weakness and abdominal pain. Bacteriological studies showed sepsis and allowed the isolation of Actinomyces odontolyticus and Abiotrophia species as causative agents. Further investigation led to a diagnosis of liver abscess from which there was also isolated Haemophilus parainfluenzae, Streptococcus intermedius and Streptococcus anginosus, as well as the above mentioned bacteria. All named microorganisms represent a part of normal oropharyngeal flora and may cause abscesses or sepsis under certain circumstances. As far as we know, liver abscess due to Abiotrophia species was never reported.

Key words: Actinomyces odontolyticus, Abiotrophia species, Haemophilus parainfluenzae, Streptococcus intermedius, Streptococcus anginosus, sepsis, liver abscess

\section{INTRODUCTION}

Actinomyces odontolyticus, Abiotrophia species, Haemophilus parainfluenzae, Streptococcus intermedius and Streptococcus anginosus represent a part of normal oropharyngeal flora and may cause abscesses or sepsis under certain circumstances. (1-4)

\section{CASE REPORT}

A 78-year-old woman presented with high fever up to $41^{\circ} \mathrm{C}$, chills and shivering of three days duration. She had no history of severe or chronic disease, and she sometimes used diclofenac due to knee pain. Her APACHE II score was 18 and her Glasgow Comma Score (GCS) was 14. Her blood pressure was $110 / 80 \mathrm{~mm} \mathrm{Hg}$, pulse $106 /$ minute, and at the cardiac apex a discreet systolic murmur could be heard. No further particular events to report from her history.

On the first day of her hospitalisation samples for urine culture and blood culture were taken. On the next day a marked deterioration of the patient's condition was observed, with recrudescence of abdominal pain. An abdominal ultrasound and CT scan showed a large liver abscess and two concrements in her gallblader. Her urine culture revealed Escherichia coli 105. A blood culture lead to the isolation of Actinomyces odontolyticus and Abiotrophia species.

Needle aspiration of the abscess was performed, pus was evacuated, and bacteriological studies allowed the isolation of the following microorganisms: Actinomyces odontolyticus, Abiotrophia species, Haemophilus parainfluenzae, Streptococcus anginosus and Streptococcus intermedius. The patient was treated with cefriaxon i.v. Clinical amelioration was noticed. Repeated ultrasound showed a small effusion around the gallbladder in communication with the abscess site.

On day 14 of her hospitalisation an opera- tive procedure was decided as an attempt for definitive recovery. A laparotomy disclosed an abscess as large as a person's fist in the subhepatic part of the lesser omentum, and subacute gallbladder changes. The gallbladder was removed and the abscess was evacuated. The postoperative course was uneventful and she was discharged home on the seventh postoperative day.

\section{DISCUSSION}

Actinomyces species are some of the commensal inhabitants of the oral cavity, gastrointestinal and urogenital tract. (1) They are filamentous, Gram-positive, anaerobic bacteria and acquire pathogenicity through invasion of breached or necrotic tissue. (5) Furthermore, actimomycosis is generally a polymicrobial infection. $(5,6)$ Of the 14 Actinomyces species, six can cause disease in humans; the most common is Actinomyces israelii, while A. odontolyticus is relatively rare. (6) Infection involving the cervicofacial area is most common (50\%) followed by abdominal involvement (20\%) and thoracic involvement (15-20\%). Actinomycosis has a worldwide distribution, affects mostly middle-aged individuals, and is two to four times more common in men. (7) Liver lesion is present in $15 \%$ of abdominal cases. (1) The infection also can spread via bloodstream to other organs, including the skin, kidney, urethras, ovaries, uterus, vertebrae, and brain. (8)

Abiotrophia species have been referred to as a nutritionally variant streptococci 
because of their fastidious nutritional requirements for growth. They are part of the normal oral, intestinal and genitourinary floras. Abiotrophia species sometimes cause sepsis, infective endocarditis, keratitis, endophtalmitis, brain abscess, sinusitis, otitis media, prostatitis, cholangitis, arthritis, osteomyelitis, and tubo-ovarial abscess. $(2,9,10)$

Haemophilus parainfluenzae is a Gramnegative, facultative anaerobe, non-motile and non-spore-forming commensal organism of the oropharynx, present in over $20 \%$ of faecal samples analysed, and has low pathogenic potential. H. parainfluenzae is an uncommon agent of human infection, but it has been found to be associated with endocarditis, bacteriemia, soft tissue infection, septic arthritis, meningitis and brain abscesses, osteomyelitis, upper respiratory tract infections and rarely liver abscess. (3)

Streptococcus anginosus and Streptococcus intermedius are the part of the Strepto- coccus anginosus group. Members of this group are Gram-positive, catalase-negative cocci, non-motile, facultative anaerobes that demonstrate variable haemolysis patterns (alpha, beta, or gamma). These organisms are part of normal flora of the human oropharynx and gastrointestinal tract with ability to cause abscesses and systemic infections. Oral, head and neck, and abdominal infections caused by members of this group are often mixed. (4)

Microorganisms that were isolated by haemoculture and by needle abscess aspiration in our patient can be found in the mouth, and bad oral hygiene, with possible damage of the mucose barrier, can lead to bacteriemia and to hepatic abscess as a consequence. All of these microorganisms are difficult to isolate, and possibly because of that not all are caught in blood cultures. As far as we know, a liver abscess due to Abiotrophia species was never reported.

\section{CONCLUSION}

In our patient sepsis was caused by rare causative agents such as Actinomyces odontolyticus and Abiotrophia species. The patient also simultaneously developed hepatic abscess, in which Haemophilus parainfluenzae, Streptococcus intermedius and Streptococcus anginosus were also isolated, in addition to the already above mentioned Actinomyces odontolyticus and Abiotrophia species. Hepatic abscess is very often caused by mixed agents, polymicrobial in its origin (as was in our case), however, Abitrophia species has never been described as a causative agent of liver abscess.

\section{ACKNOWLEDGMENT}

We thank the staff of University Hospital Dubrava.

\section{REFERENCES}

1. Uehara Y, Takahashi T, Yagoshi M et al. Liver abscess of Actinomyces israelii in a hemodialysis patient: case report and review of the literature. Inter Med 2010; 49: 2017- 2020.

2. Senn L, Entenza JM, Greub G et al. Bloodstream and endovascular infections due to Abiotrophia defectiva and Granulicatella species. BMC Infect Dis 2006; 6: 9.

3. Friedl J, Stift A, Berlakovich GA et al. Haemophilus parainfluenzae liver abscess after successful liver transplantation. J Clin Microbiol 1998; 818- 819.

4. Stratton CW. Infections due to the Streptococcus anginosus (Streptococcus milleri) group. [last updated March 24, 2015., cited May 09, 2015.]. Available from: http://www.uptodate.com/contents/infections-due-to-the-streptococcus-anginosus-streptococcusmilleri group?source=search_result\&search=streptococcus+anginosus\&selectedTitle $=1 \sim 18$.

5. Okulitz JF. Actinomycosis. [updated September 25, 2015., cited May 09, 2015.]. Available from: http://emedicine.medscape.com/ article/211587-overview.

6. Alekh K, Dazley J, Sison R, Slim J, Boghossian J. A rare case of Actinomyces israelii bacteriemia. JMM Case Reports 2014; doi 10.1099jmmcr.0.000158.

7. Wong Kee Song LM, Marcon NE. Abdominal actinomycosis. [last updated: July 15, 2014., cited May 09, 2015.]. Available from: http://www.uptodate.com/contents/abdominal-actinomycosis?source=search_result\&search=abdominal+actinomycosis\&selecte dTitle $=1 \sim 1$.

8. Medical Disability Advisor. Actinomycosis. [cited May 09, 2015.].Available from: http://www.mdguidelines.com/actinomycosis.

9. Uehara K, Chikuda H, Higurashi Y etal. Pyogenic discitis due to Abiotrophia adiacens. Int J Surg Case Rep 2013; 4: 1107- 1109.

10. Gensheimer WG, Reddy SY, Mulconry M, Greves C. Abiotrophia/Granulicatellatubo- ovarian abscess in an adolescent virginal female. J Pediatr Adolesc Gynecol 2010; 23: e9- 12. 